\title{
Commentary: More is more: Root stabilization for regurgitant aortic valve repair
}

\author{
T. Brett Reece, MD, and Muhammad Aftab, MD
}

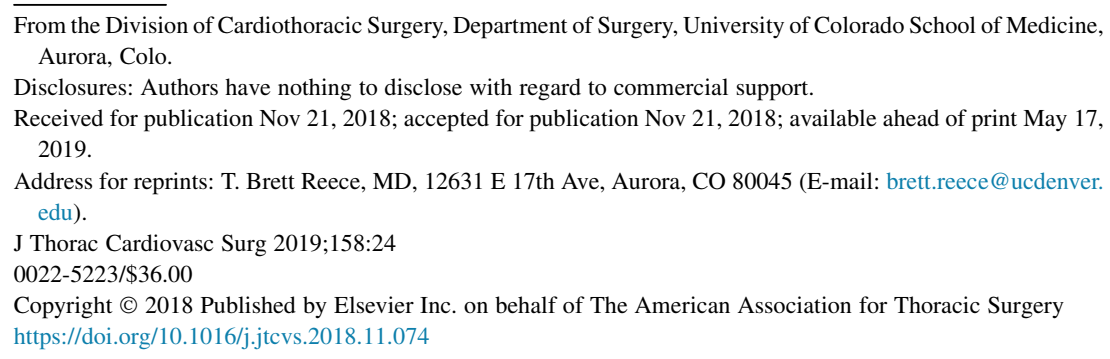

Most discussions regarding the optimal valve for replacement include the disclaimer that a perfect replacement does not exist today. The argument of mechanical anticoagulation versus tissue durability continues, despite advances in technique and technology. Since David and Feindel ${ }^{1}$ originally described the valve-sparing root procedure, the viability of aortic valve repair has become apparent. Discussion in valve repair forums, such as the article in this issue of the Journal by Mastrobuoni and colleagues ${ }^{2}$ from the Brussels group, have demonstrated that true, durable salvation of the aortic valve is not only feasible but also durable, with and without root dilation. As experience has been gained, the concept of the root being part of the valve function has widened. The evolutions of valve-sparing root repair and aortic valve repair have pushed the limits of indication, including raising discussion of whether a root procedure could be indicated for isolated aortic valve pathology. Transcatheter valve replacement has probably stifled the evolution of this idea in the United States because of worry about the need for open reoperation in the absence of a transcatheter indication for aortic regurgitation. As such, most surgeons would end up placing a tissue valve in most patients, expecting the durability to be overcome by the availability of future valve-in-valve replacement. The lack of a percutaneous bailout for valve preservation has stifled widespread adoption of this approach.

The root stabilization from a valve-sparing root procedure has become an effective, durable solution. Although the technique is still not to the point of widespread acceptance, the article of Mastrobuoni and colleagues ${ }^{2}$ demonstrates the use of root replacement as an effective part of aortic valve repair, addressing dilated but nonaneurysmal segments of the annulus, sinus, and sinotubular junction. Addressing these issues has become standard for root pathologies, but it appears to critical for achieving valve competence with time. Opponents of the root stabilization are quick to point on increased risks of a root procedure

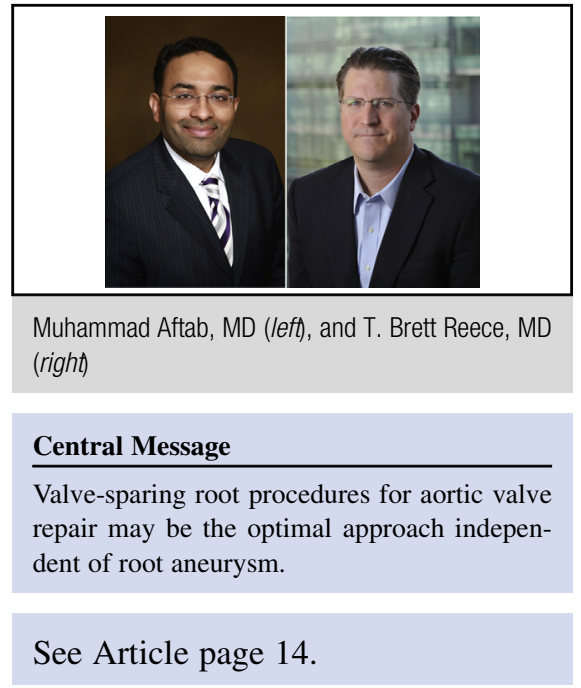

relative to an isolated valve procedure. The article of Mastrobuoni and colleagues ${ }^{2}$ addresses this concern by demonstrating an incredibly low mortality for the more complex procedure. It demonstrates that aortic valve repair with root stabilization is safe and durable, even relative to conventional aortic valve replacement results. The aggressive approach may be justified by both short-term and midterm outcomes.

In conclusion, the results of valve-sparing root procedures presented by Mastrobuoni and colleagues ${ }^{2}$ are to be applauded. Further, the demonstration of the safety and efficacy of addressing the root to save valve function deserves careful consideration to preserve aortic valve function longitudinally. The paradigm of transcatheter bailout of a tissue valve should be questioned when an alternative exists for regurgitant valves. The importance of salvaging valve durability should not be compromised by incomplete valve repair. As more experience accumulates and shortterm outcomes demonstrate comparative effectiveness, valve-sparing root procedures may emerge as the most complete option for approaching aortic valve repair independent of a root indication for replacement.

\section{References}

1. David TE, Feindel CM. An aortic valve-sparing operation for patients with aortic incompetence and aneurysm of the ascending aorta. J Thorac Cardiovasc Surg. 1992; 103:617-21; discussion 622.

2. Mastrobuoni S, de Kerchove L, Navarra E, Watremez C, Vancraeynest D, Rubay J, et al. Long-term experience with valve-sparing reimplantation technique for the treatment of aortic aneurysm and aortic regurgitation. J Thorac Cardiovasc Surg. 2019;158:14-23. 